\title{
A Closed-Form Observer for the Channel Flow Navier-Stokes System
}

\author{
Rafael Vazquez and Miroslav Krstic
}

\begin{abstract}
The observer design for non-discretized NavierStokes partial differential equations has so far been an open problem. We present a nonlinear PDE observer that estimates the velocity and pressure fields for an infinite channel flow. Also know as the Poisseuille flow, this problem is frequently cited as a paradigm for transition to turbulence, and is a benchmark for flow control and turbulence estimation. Our observer consists of a copy of the nonlinear Navier-Stokes equations, combined with linear injection of output estimation error, with observer gains designed in closed form using backstepping. Pressure and skin friction at one of the walls are the only quantities needed for measurement. For a fully developed channel flow (whether laminar or turbulent), with a Reynolds number possibly above the critical value, but not too far above it, the observer is guaranteed to be convergent to the actual velocity and pressure field, provided its initial estimates are not too far from the actual initial profile. An output feedback result for the linearized infinite channel flow is also presented, combining the observer in this paper with a controller presented in a companion CDC'05 paper. Both results are presented for the $2 \mathrm{D}$ case for clarity of exposition, however an extension to $3 \mathrm{D}$ is straightforward.
\end{abstract}

\section{INTRODUCTION}

The absence of effective state estimators for Navier-Stokes equations modeling turbulent fluid flows is considered one of the key obstacles to reliable, model-based weather forecasting. We present a nonlinear observer for the channel flow benchmark problem. The observer measures only pressure and skin friction at one of the walls of the channel and estimates velocities and pressure throughout the channel. Previous observer designs for the channel flow were in the form of an Extended Kalman Filter for the spatially discretized Navier-Stokes equations and employed highdimensional algebraic Riccati equations for computation of observer gains [2]. The structure of our observer is similar to an Extended Kalman Filter, however it is designed for the continuum Navier-Stokes model and the output injection gains are are given explicitly, by a symbolically computable formula.

For notational clarity the result is presented in $2 \mathrm{D}$. It extends to $3 \mathrm{D}$ in a straightforward way due to spatial invariance in the spanwise direction.

We start the paper stating the mathematical model of the problem, which is the fully nonlinear incompressible Navier-Stokes PDE (Section II). In Section III we introduce the nonlinear observer for estimation of the velocity and pressure fields, with explicit expressions for the output injection gains. Section IV deals with the proof of local convergence for the observer error system. Fourier transform allows separate analysis for different wave numbers. For some wave numbers, a combination of output injection and a

This work was supported by NSF grant number CMS-0329662.

Department of Mechanical and Aerospace Engineering, University of California at San Diego

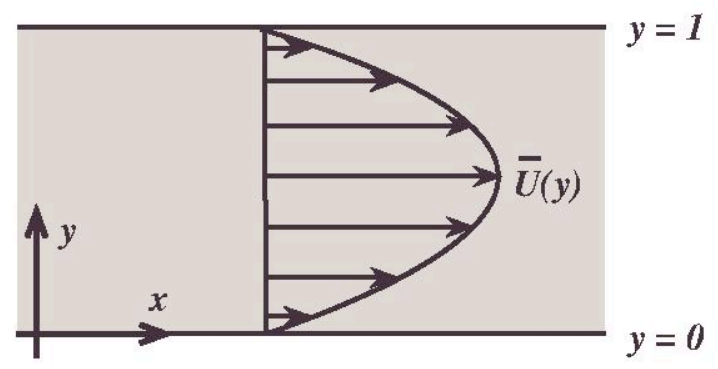

Fig. 1. 2D Channel Flow and equilibrium profile

stabilizing term found through a backstepping transformation guarantee observer convergence. Finding the kernel of the transformation is the design task. We derive an integral equation which is used to symbolically compute the observer gains. For the very large and the very small wave numbers the system is known to be exponentially stable, and simply a copy of the plant is used. These two results are combined to prove observer error convergence for all wave numbers. Section V presents a complete output feedback design combining the results of the present paper with control results in a companion paper [7].

\section{MODEL}

Consider a 2D incompressible channel flow evolving in a semi-infinite rectangle $(x, y) \in(-\infty, \infty) \times[0,1]$ like shown in Figure 1. The dimensionless velocity field is governed by the Navier-Stokes equations

$$
\begin{aligned}
U_{t} & =\frac{1}{R e}\left(U_{x x}+U_{y y}\right)-U U_{x}-V U_{y}-P_{x}, \\
V_{t} & =\frac{1}{R e}\left(V_{x x}+V_{y y}\right)-U V_{x}-V V_{y}-P_{y},
\end{aligned}
$$

and the continuity equation

$$
U_{x}+V_{y}=0,
$$

where $U$ denotes the streamwise velocity, $V$ the wall-normal velocity, $P$ the pressure, and $R e$ is the Reynolds number. Instead of using (3) we can derive a Poisson equation that $P$ verifies, just combining (1), (2) and (3)

$$
P_{x x}+P_{y y}=-2\left(V_{y}\right)^{2}-2 V_{x} U_{y} .
$$

The boundary conditions verified by (1), (2) and (4) are

$$
\begin{aligned}
U(t, x, 0) & =U(t, x, 1)=0, \\
V(t, x, 0) & =V(t, x, 1)=0, \\
P_{y}(t, x, 0) & =\frac{1}{R e} U_{x y}(t, x, 0), \\
P_{y}(t, x, 1) & =\frac{1}{R e} U_{x y}(t, x, 1),
\end{aligned}
$$

where (7)-(8) are deduced from (2) and (3). 


\section{OBSERVER}

The observer consists on a copy of (1)-(2), to which we add output injection of the pressure $P$ and the streamwise velocity gradient $U_{y}$ (proportional to friction) at the wall

$$
\begin{aligned}
\hat{U}_{t}= & \frac{1}{R e}\left(\hat{U}_{x x}+\hat{U}_{y y}\right)-\hat{P}_{x}-\hat{U} \hat{U}_{x}-\hat{V} \hat{U}_{y} \\
& -\frac{1}{\operatorname{Re}} \int_{-\infty}^{\infty} Q_{1}(x-\xi, y)\left(U_{y}(\xi, 0)-\hat{U}_{y}(\xi, 0)\right) d \xi \\
& +\int_{-\infty}^{\infty} Q_{2}(x-\xi, y)(P(\xi, 0)-\hat{P}(\xi, 0)) d \xi \\
\hat{V}_{t}= & \frac{1}{\operatorname{Re}}\left(\hat{V}_{x x}+\hat{V}_{y y}\right)-\hat{P}_{y}-\hat{U} \hat{V}_{x}-\hat{V} \hat{V}_{y} \\
& -\frac{1}{\operatorname{Re}} \int_{-\infty}^{\infty} \hat{V}_{y}(\xi, 0) \int_{-\infty}^{\infty} \chi(k) l(k, y, 0) \mathrm{e}^{2 \pi i k(x-\xi)} d k d \xi \\
& +\frac{1}{\operatorname{Re}} \int_{-\infty}^{\infty} Q_{2}(x-\xi, y)\left(U_{y}(\xi, 0)-\hat{U}_{y}(\xi, 0)\right) d \xi \\
& +\int_{-\infty}^{\infty} Q_{1}(x-\xi, y)(P(\xi, 0)-\hat{P}(\xi, 0)) d \xi,
\end{aligned}
$$

where as usual the observer (estimated) variables are denoted by a hat. The pressure $\hat{P}$ is governed by a copy of (4)

$$
\hat{P}_{x x}+\hat{P}_{y y}=-2\left(\hat{V}_{y}\right)^{2}-2 \hat{V}_{x} \hat{U}_{y},
$$

and the boundary conditions for the observer are

$$
\begin{aligned}
\hat{U}(t, x, 0) & =\hat{U}(t, x, 1)=0, \\
\hat{V}(t, x, 0) & =\hat{V}(t, x, 1)=0, \\
\hat{P}_{y}(t, x, 0) & =\frac{-1}{\operatorname{Re}} \hat{U}_{x y}(t, x, 0), \\
\hat{P}_{y}(t, x, 1) & =\frac{-1}{R e} \hat{U}_{x y}(t, x, 1) .
\end{aligned}
$$

The observer employs two output injection kernels defined

$$
\begin{aligned}
& Q_{1}(x, y)=R_{1}(x, y, M)-R_{1}(x, y, m), \\
& Q_{2}(x, y)=R_{2}(x, y, M)-R_{2}(x, y, m),
\end{aligned}
$$

where

$$
\begin{aligned}
R_{1}(x, y, k)= & \frac{2 x}{\pi\left(x^{2}+y^{2}\right)^{2}}[y \cosh (2 \pi k y) \sin (2 \pi k x) \\
& -x \sinh (2 \pi k y) \cos (2 \pi k x)] \\
& -\frac{2 k}{\left(x^{2}+y^{2}\right)}[y \cosh (2 \pi k y) \cos (2 \pi k x) \\
& +x \sinh (2 \pi k y) \sin (2 \pi k x)] \\
& +\frac{\sinh (2 \pi k y) \cos (2 \pi k x)}{\pi\left(x^{2}+y^{2}\right)}, \\
R_{2}(x, y, k)= & \frac{2 x}{\pi\left(x^{2}+y^{2}\right)^{2}}[x \cosh (2 \pi k y) \sin (2 \pi k x) \\
& +y \sinh (2 \pi k y) \cos (2 \pi k x)] \\
& -\frac{2 k}{\left(x^{2}+y^{2}\right)}[x \cosh (2 \pi k y) \cos (2 \pi k x) \\
& -y \sinh (2 \pi k y) \sin (2 \pi k x)] \\
& -\frac{\cosh (2 \pi k y) \sin (2 \pi k x)}{\pi\left(x^{2}+y^{2}\right)}
\end{aligned}
$$

$Q_{1}$ is shown in Fig. 2, and $Q_{2}$ has a very similar shape. In equation (10) there is also an additional term,

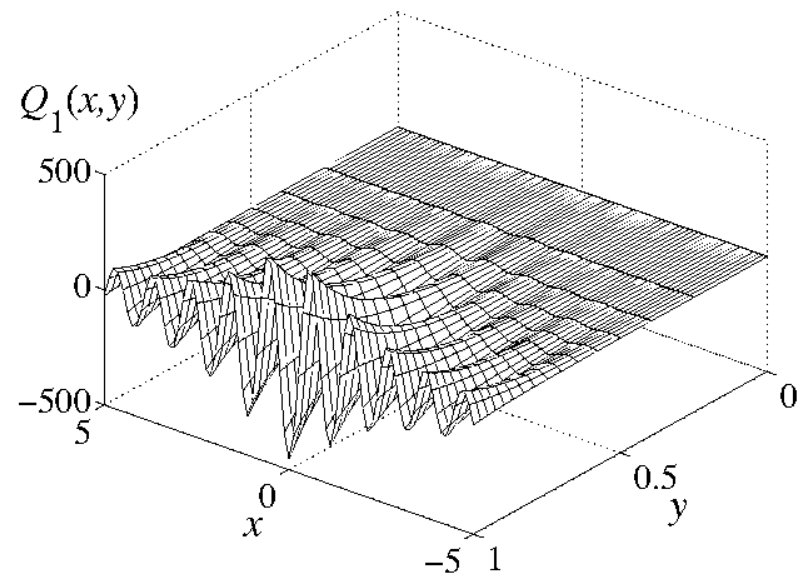

Fig. 2. $Q_{1}(x, y)$

$\frac{1}{R e} \int_{-\infty}^{\infty} \chi(k) l(k, y, 0) \mathrm{e}^{2 \pi i k(x-\xi)} d k$. The purpose of this gain is stabilization of the observer error equation and it is also a form of output injection, because by continuity $V_{y}(x, 0)$ is known to be zero. Therefore, the value of $\hat{V}_{y}(x, 0)$, which should be zero if the observer has converged to the real value, measures a discrepancy between the observer and the real system, which is fed back. For this term,

$$
\chi(k)=\left\{\begin{array}{cc}
1, & m \leq|k| \leq M \\
0, & \text { otherwise }
\end{array}\right.
$$

where $m=\frac{1}{32 \pi R e}$ and $M=\frac{1}{\pi} \sqrt{\frac{R e}{2}}$, and

$$
l(k, y, \eta)=\lim _{n \rightarrow \infty} l_{n}(k, y, \eta)
$$

is recursively defined as 1

$$
\begin{aligned}
l_{0}= & -\frac{\operatorname{Re}}{3} \pi i k(1-y)\left((1-y)^{2}+3 \eta^{2}-3\right) \\
& +4 i \operatorname{Rey}(y-1) \sinh (2 \pi k(y-\eta)) \\
& +2(1-y) i \frac{\operatorname{Re}}{\pi k}(1-\cosh (2 \pi k(y-\eta))) \\
l_{n}= & l_{n-1} \\
& +8 \operatorname{Re} \pi k i \int_{y-\eta}^{2-(y+\eta)} \int_{0}^{y-\eta} \int_{-\delta}^{\delta} \cosh (\pi k(\xi+\delta)) \\
& \times(\gamma-\delta-1) l_{n-1}\left(k, \frac{\gamma+\delta}{2}, \frac{\gamma+\xi}{2}\right) d \xi d \delta d \gamma \\
& +\frac{\operatorname{Re}}{2} \pi i k \int_{y-\eta}^{2-(y+\eta)} \int_{0}^{y-\eta}(\gamma+\delta-2) \\
& \times(\gamma+\delta) l_{n-1}\left(k, \frac{\gamma+\delta}{2}, \frac{\gamma-\delta}{2}\right) d \delta d \gamma
\end{aligned}
$$

The terms of this recursion can be computed symbolically as they only involve integration of polynomials and exponentials. In implementation, only a few steps will be sufficient to obtain a highly accurate approximation because the recursion is rapidly convergent [5]. The first order approximation

$$
l_{0}(x, y, 0)=\int_{-\infty}^{\infty} \chi(k) l_{0}(k, y, 0) \mathrm{e}^{2 \pi i k(x-\xi)} d k
$$

\footnotetext{
${ }^{1}$ This recursion is proved convergent, smooth, and uniformly bounded
} over $(y, \eta) \in[0,1]^{2}$, with a bound continuously dependent on $k$. 


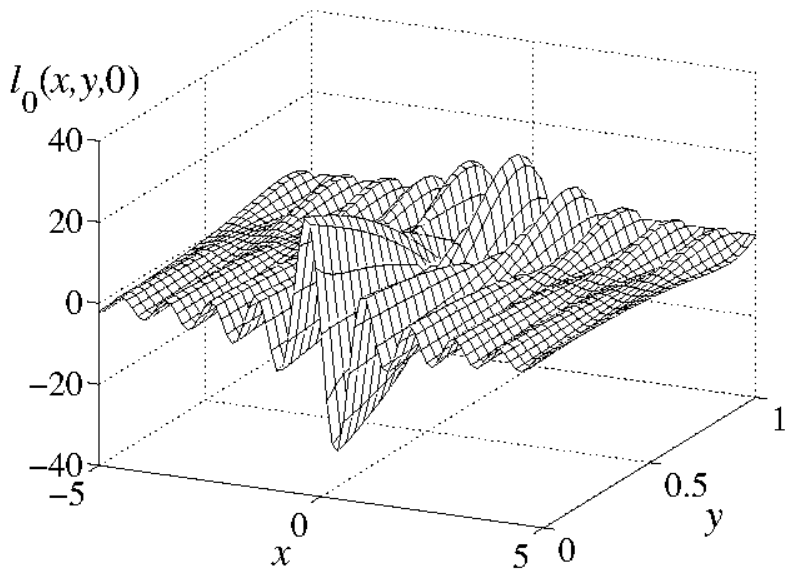

Fig. 3. $l_{0}(x, y, 0)$

is plotted in Fig. 3. Comparing this gain with the previously defined $Q_{1}$ and $Q_{2}$ gains, it can be seen that the output injections of $P(x, 0)$ and $U_{y}(x, 0)$ are important away from the measurements and near the $y=1$ boundary, while the term that accounts for the discrepancy in $\hat{V}_{y}(x, 0)$ is bigger near the $y=0$ boundary.

Remark 1: The output injection operators in the observer are spatially invariant in the $x$ direction in the sense of [1]. ${ }^{2}$ This is due to the fact that the plant itself is invariant in the $x$ direction. This is very significant since it allows an easier design by exploiting the structure of the plant. It also makes possible a straightforward extension to a $3 \mathrm{D}$ result, for the case of a channel infinite in two directions, with the new direction $z$ being also of spatial invariance.

Remark 2: It should be noted that the observer estimated "velocity field" $(\hat{U}, \hat{V})$ is not required to verify the incompressibility condition (3). In fact, it can be shown from equations (9)-(15) that as the estimated velocity field evolves in time, its divergence will also verify an evolution equation which will make it change, even if the initial condition for the observer is a divergence-free velocity field. This is not really a problem, since the proof shows that the observer error converges to zero and therefore the observer converges to the real, divergence-free velocity field. The transient value of the estimated field is allowed to have nonzero divergence as an extra degree of freedom.

\section{OB SERVER CONVERGENCE Proof}

As common for infinite channels, we use a Fourier transform in $x$. The transform pair (direct and inverse transform) has the following definition

$$
\begin{aligned}
& f(k, y)=\mathcal{F}\{f(x, y)\}=\int_{-\infty}^{\infty} f(x, y) \mathrm{e}^{-2 \pi i k x} d x, \\
& f(x, y)=\mathcal{F}^{-1}\{f(k, y)\}=\int_{-\infty}^{\infty} f(k, y) \mathrm{e}^{2 \pi i k x} d x
\end{aligned}
$$

Note that we use the same symbol $f$ for both the original $f(x, y)$ and the image $f(k, y)$. In hydrodynamics $k$ is referred to as the "wave number." The output injection kernels

\footnotetext{
${ }^{2}$ The feedback operator commutes with the translation in the $x$ direction.
}

defined in (16) and (17) written in Fourier space are

$$
\begin{aligned}
& Q_{1}(k, y)=\chi(k) 2 \pi k \sinh (2 \pi k y), \\
& Q_{2}(k, y)=\chi(k) 2 \pi k i \cosh (2 \pi k y),
\end{aligned}
$$

where we have used the truncating function $\chi(k)$. The truncation for large wave numbers doesn't imply a loss of smoothness in physical space, as evident from the smooth expressions (16)-(19).

Substracting the observer equations from the plant equations we obtain the error equations, with states $\tilde{U}=U-$ $\hat{U}, \tilde{V}=V-\hat{V}, \tilde{P}=P-\hat{P}$. Note that from continuity, $V_{y}(x, 0)=0$ and therefore $\tilde{V}_{y}(x, 0)=-\hat{V}_{y}(x, 0)$.

First we eliminate the pressure error, whose equation in Fourier space is

$$
\begin{aligned}
-4 \pi^{2} k^{2} \tilde{P}+\tilde{P}_{y y}= & 2 \mathcal{F}\left\{\left(\hat{V}_{y}\right)^{2}-\left(V_{y}\right)^{2}\right. \\
& \left.+\hat{V}_{x} \hat{U}_{y}-V_{x} U_{y}\right\}, \\
\tilde{P}_{y}(k, 0)= & \frac{2 \pi k i}{R e} \tilde{U}_{y}(k, 0), \\
\tilde{P}_{y}(k, 1)= & \frac{2 \pi k i}{R e} \tilde{U}_{y}(k, 1) .
\end{aligned}
$$

The solution to this equation is

$$
\begin{aligned}
\tilde{P}= & \int_{0}^{y} \frac{\sinh (2 \pi k(y-\eta))}{\pi k} \mathcal{F}\left\{\left(\hat{V}_{y}\right)^{2}-\left(V_{y}\right)^{2}\right. \\
& \left.+\hat{V}_{x} \hat{U}_{y}-V_{x} U_{y}\right\}(k, \eta) d \eta \\
& -\frac{\cosh (2 \pi k y)}{\sinh (2 \pi k)} \int_{0}^{1} \frac{\cosh (2 \pi k(1-\eta))}{\pi k} \mathcal{F}\left\{\left(\hat{V}_{y}\right)^{2}\right. \\
& \left.-2\left(V_{y}\right)^{2}+\hat{V}_{x} \hat{U}_{y}-V_{x} U_{y}\right\}(k, \eta) d \eta \\
& -\frac{i}{R e}\left[\frac{\cosh (2 \pi k y)}{\sinh (2 \pi k)} \tilde{U}_{y}(k, 1)+(\sinh (2 \pi k y)\right. \\
& \left.\left.-\cosh (2 \pi k y) \frac{\cosh (2 \pi k)}{\sinh (2 \pi k)}\right) \tilde{U}_{y}(k, 0)\right],
\end{aligned}
$$

and noting that

$$
\begin{aligned}
\tilde{P}(k, 0)= & \frac{-1}{\sinh (2 \pi k)}\left(\int _ { 0 } ^ { 1 } \frac { \operatorname { c o s h } ( 2 \pi k ( 1 - \eta ) ) } { \pi k } \mathcal { F } \left\{\left(\hat{V}_{y}\right)^{2}\right.\right. \\
& \left.-2\left(V_{y}\right)^{2}+\hat{V}_{x} \hat{U}_{y}-V_{x} U_{y}\right\}(k, \eta) d \eta \\
& \left.+\frac{i}{R e}\left[\tilde{U}_{y}(k, 1)-\cosh (2 \pi k) \tilde{U}_{y}(k, 0)\right]\right),(33)
\end{aligned}
$$

we can write the pressure error as

$$
\begin{aligned}
\tilde{P}= & \int_{0}^{y} \frac{\sinh (2 \pi k(y-\eta))}{\pi k} \mathcal{F}\left\{\left(\hat{V}_{y}\right)^{2}-\left(V_{y}\right)^{2}\right. \\
& \left.+\hat{V}_{x} \hat{U}_{y}-V_{x} U_{y}\right\}(k, \eta) d \eta \\
& +\cosh (2 \pi k y) \tilde{P}(k, 0) \\
& -\frac{i}{R e} \sinh (2 \pi k y) \tilde{U}_{y}(k, 0) .
\end{aligned}
$$

We define the fluctuation variable

$$
u(t, x, y)=U(t, x, y)-\bar{U}(y),
$$

where $\bar{U}(y)=4 y(1-y)$ is the parabolic equilibrium profile of the channel flow, shown in Fig. 1. 
Using (34) for eliminating the pressure, and the Fourier transforms for the output injection kernels (27)-(28), the velocity error equations expressed in Fourier space are

$$
\begin{aligned}
\tilde{U}_{t}= & \frac{1}{R e}\left(-4 \pi^{2} k^{2} \tilde{U}+\tilde{U}_{y y}\right)+8 i \pi k y(y-1) \tilde{U} \\
& +4(2 y-1) \tilde{V} \\
& +16 \pi k \int_{0}^{y} \sinh (2 \pi k(y-\eta))(2 \eta-1) \tilde{V}(k, \eta) d \eta \\
& -(1-\chi(k)) 2 i \pi k \cosh (2 \pi k y) \tilde{P}(k, 0) \\
& -(1-\chi(k)) \frac{2 \pi k}{R e} \sinh (2 \pi k y) \tilde{U}_{y}(k, 0) \\
& +\mathcal{N}_{U}(\tilde{V}, \tilde{U}, u, V), \\
\tilde{V}_{t}= & \frac{1}{R e}\left(-4 \pi^{2} k^{2} \tilde{V}+\tilde{V}_{y y}\right)+8 i \pi k y(y-1) \tilde{V} \\
& -16 \pi k i \int_{0}^{y} \cosh (2 \pi k(y-\eta))(2 \eta-1) \tilde{V}(k, \eta) d \eta \\
& -(1-\chi(k)) 2 \pi k \sinh (2 \pi k y) \tilde{P}(k, 0) \\
& +(1-\chi(k)) \frac{2 \pi k i}{R e} \cosh (2 \pi k y) \tilde{U}_{y}(k, 0) \\
& -\chi(k) \frac{1}{R e} l(k, y, 0) \tilde{V}_{y}(k, 0) \\
& +\mathcal{N}_{V}(\tilde{V}, \tilde{U}, u, V),
\end{aligned}
$$

where

$$
\begin{aligned}
\tilde{P}(k, 0)= & -\frac{8 i}{\sinh (2 \pi k)} \int_{0}^{1} \cosh (2 \pi k(1-\eta)) \\
& \times(2 \eta-1) \tilde{V}(k, \eta) d \eta \\
& +\mathcal{N}_{P}(\tilde{V}, \tilde{U}, u, V)
\end{aligned}
$$

with boundary conditions

$$
\begin{aligned}
& \tilde{U}(t, x, 0)=\tilde{U}(t, x, 1)=0 \\
& \tilde{V}(t, x, 0)=\tilde{V}(t, x, 1)=0
\end{aligned}
$$

and higher order terms

$$
\begin{aligned}
\mathcal{N}_{U}= & \mathcal{F}\left\{\tilde{U} \tilde{U}_{x}-\tilde{U} u_{x}-u \tilde{U}_{x}+\tilde{V} \tilde{U}_{y}-\tilde{V} u_{y}-V \tilde{U}_{y}\right\} \\
& +2 i \int_{0}^{y} \sinh (2 \pi k(1-\eta)) \mathcal{F}\left\{2 \tilde{V}_{y}(x, \eta) V_{y}(x, \eta)\right. \\
& -\left(\tilde{V}_{y}\right)^{2}(x, \eta)+\tilde{V}_{x}(x, \eta) u_{y}(x, \eta) \\
& \left.+V_{x}(x, \eta) \tilde{U}_{y}(x, \eta)-\tilde{V}_{x}(x, \eta) \tilde{U}_{y}(x, \eta)\right\} d \eta \\
\mathcal{N}_{V}= & \mathcal{F}\left\{\tilde{U}_{x}-\tilde{U} V_{x}-u \tilde{V}_{x}+\tilde{V} \tilde{V}_{y}-\tilde{V} V_{y}-V \tilde{V}_{y}\right\} \\
& +2 \int_{0}^{y} \cosh (2 \pi k(1-\eta)) \mathcal{F}\left\{2 \tilde{V}_{y}(x, \eta) V_{y}(x, \eta)\right. \\
& -\left(\tilde{V}_{y}\right)^{2}(x, \eta)+\tilde{V}_{x}(x, \eta) u_{y}(x, \eta) \\
& \left.+V_{x}(x, \eta) \tilde{U}_{y}(x, \eta)-\tilde{V}_{x}(x, \eta) \tilde{U}_{y}(x, \eta)\right\} d \eta,(42) \\
\mathcal{N}_{P}= & \frac{1}{\pi k \sinh (2 \pi k)} \int_{0}^{1} \cosh (2 \pi k(1-\eta)) \\
& \times \mathcal{F}\left\{2 \tilde{V}_{y}(x, \eta) V_{y}(x, \eta)-\left(\tilde{V}_{y}\right)^{2}(x, \eta)\right. \\
& +\tilde{V}_{x}(x, \eta) u_{y}(x, \eta)+V_{x}(x, \eta) \tilde{U}_{y}(x, \eta) \\
& \left.-\tilde{V}_{x}(x, \eta) \tilde{U}_{y}(x, \eta)\right\} d \eta
\end{aligned}
$$

which are quadratic in $\tilde{V}, \tilde{U}, u, V$.

When the observer state $(\hat{U}, \hat{V})$ is close to the actual state $(U, V)$, and when the fluctuation $(u, V)$ around the equilibrium state is small, then $\mathcal{N}_{U}(\tilde{U}, \tilde{V}, u, V), \mathcal{N}_{V}(\tilde{U}, \tilde{V}, u, V)$ and $\mathcal{N}_{P}(\tilde{U}, \tilde{V}, u, V)$ are small and dominated by the linear terms in the equations. The linearization of the subsystem (with $\mathcal{N}_{U}, \mathcal{N}_{V}$, and $\mathcal{N}_{P}$ neglected) is uncoupled for each wave number, and therefore can be studied separately for $m \leq|k| \leq M$, which we will refer as the observed wave number range, and $|k|>M,|k|<m$, the unobserved wave number range, where $m$ and $M$ are parameters that will be defined to ensure stability for the unobserved wave number equations.

\section{A. Observed wave number analysis}

For $m \leq|k| \leq M$, the output injection kernels eliminate the presence of $\tilde{P}(k, 0)$ and $\tilde{U}_{y}(k, 0)$. The linearized plant is

$$
\begin{aligned}
\tilde{U}_{t}= & \frac{1}{R e}\left(-4 \pi^{2} k^{2} \tilde{U}+\tilde{U}_{y y}\right)+8 i \pi k y(y-1) \tilde{U} \\
& +(2 y-1) \tilde{V}+16 \pi k \int_{0}^{y} \sinh (2 \pi k(y-\eta)) \\
& \times(2 \eta-1) \tilde{V}(k, \eta) d \eta, \\
\tilde{V}_{t}= & \frac{1}{\operatorname{Re}}\left(-4 \pi^{2} k^{2} \tilde{V}+\tilde{V}_{y y}\right)+8 i \pi k y(y-1) \tilde{V} \\
& -16 \pi k i \int_{0}^{y} \cosh (2 \pi k(y-\eta))(2 \eta-1) \tilde{V}(k, \eta) d \eta \\
& -\frac{1}{R e} l(k, y, 0) \tilde{V}_{y}(k, 0),
\end{aligned}
$$

with boundary conditions (39)-(40). Equation (44) is exponentially stable when $\tilde{V}=0$. Thus for $\tilde{U}(t)$ to be locally convergent, it is enough to ensure that $\vec{V}$ goes to zero. Therefore we have to stabilize the $\tilde{V}$ subsystem. For this we use the gain $l(k, y, 0)$, which is designed using the backstepping observer design technique [6] by transforming $\tilde{V}$ equation for $m \leq|k| \leq M$ into the family of heat equations (parametrized by $k$ )

$$
\begin{aligned}
\alpha_{t} & =\frac{1}{R e}\left(-4 \pi^{2} k^{2} \alpha+\alpha_{y y}\right) \\
\alpha(k, 0) & =0 \\
\alpha(k, 1) & =0
\end{aligned}
$$

where

$$
\tilde{V}=\alpha-\int_{0}^{y} l(k, y, \eta) \alpha(t, k, \eta) d \eta
$$

is the backstepping transformation as defined in [6]. The kernel $l$ is found to verify the following equation

$$
\begin{aligned}
\frac{1}{R e} l_{\eta \eta}= & \frac{1}{R e} l_{y y}+8 \pi i k y(y-1) l \\
& -16 \pi k i(2 \eta-1) \cosh (2 \pi k(y-\eta)) \\
& +16 \pi k i \int_{\eta}^{y}(2 \xi-1) \cosh (2 \pi k(y-\xi)) \\
& \times l(k, \xi, \eta) d \xi,
\end{aligned}
$$

a hyperbolic partial integro-differential equation in the region $\mathcal{T}=\{(y, \eta): 0 \leq y \leq 1,0 \leq \eta \leq y\}$ with boundary 
conditions

$$
\begin{aligned}
& l(y, y)=-\frac{2 R e}{3} \pi i k y^{2}(2 y-3), \\
& l(1, \eta)=0 .
\end{aligned}
$$

This equation can be transformed into an integral equation from which the explicit solution given in (22)-(23) is obtained, following the steps outlined in [6]. Estimating the solution of the integral equation one gets the following result.

Theorem 1: The equation (50) with boundary conditions (51)-(52) has a unique $\mathcal{C}^{2}(\mathcal{T})$ solution analytic on $k$, expressed by series (22)-(23).

The proof uses the same argument as in [5], slightly modified to account for a complex-valued kernel and using the fact that the terms are analytic in $k$. See [7] for an extended explanation.

Once the kernel $l$ is found, the gain that appears in (45) is explicitly known and guarantees exponential stability of $\tilde{V}$, and therefore of $\tilde{U}$, for all $m \leq|k| \leq M$.

\section{B. Unobserved wave number analysis}

When $|k|>M$ or $|k|<m$, the observer error linearization verifies the following equations

$$
\begin{aligned}
\tilde{U}_{t}= & \frac{1}{R e}\left(-4 \pi^{2} k^{2} \tilde{U}+\tilde{U}_{y y}\right)+8 i \pi k y(y-1) \tilde{U} \\
& +16 \pi k \int_{0}^{y} \sinh (2 \pi k(y-\eta))(2 \eta-1) \tilde{V}(k, \eta) d \eta \\
& +(2 y-1) \tilde{V}-2 i \pi k \cosh (2 \pi k y) \tilde{P}(k, 0) \\
& -\frac{2 \pi k}{R e} \sinh (2 \pi k y) \tilde{U}_{y}(k, 0), \\
\tilde{V}_{t}= & \frac{1}{R e}\left(-4 \pi^{2} k^{2} \tilde{V}+\tilde{V}_{y y}\right)+8 i \pi k y(y-1) \tilde{V} \\
& -16 \pi k i \int_{0}^{y} \cosh (2 \pi k(y-\eta))(2 \eta-1) \tilde{V}(k, \eta) d \eta \\
& -2 \pi k \sinh (2 \pi k y) \tilde{P}(k, 0) \\
& +\frac{2 \pi k i}{R e} \cosh (2 \pi k y) \tilde{U}_{y}(k, 0),
\end{aligned}
$$

where

$$
\begin{aligned}
\tilde{P}(k, 0)= & -\frac{8 i}{\sinh (2 \pi k)} \int_{0}^{1} \cosh (2 \pi k(1-\eta)) \\
& \times(2 \eta-1) \tilde{V}(k, \eta) d \eta
\end{aligned}
$$

with boundary conditions (39)-(40). These are just the linearized Navier-Stokes equations. Then, following exactly the same arguments as in [7] for the uncontrolled Navier-Stokes equations, choosing $m=\frac{1}{32 \pi R e}$ and $M=\frac{1}{\pi} \sqrt{\frac{R e}{2}}$ stability of the unobserved wave number range is guaranteed.

\section{Analysis for the entire wave number range}

The analysis sketched in the previous section can be combined for all wave numbers, to prove the following result.

Theorem 2: Consider the system (1), (2) and (4), with boundary conditions (5)-(8), and the system (9)-(15), and suppose that both have classical solutions. Consider now the observer error system. There exists positive constants $C_{1}$ and
$C_{2}$ such that, if the $L_{2}$ norms of the initial conditions for $\tilde{U}$ and $\tilde{V}$ are less than $C_{1}$, i.e.

$$
\int_{0}^{1} \int_{-\infty}^{\infty}\left(\tilde{U}^{2}(0, x, y)+\tilde{V}^{2}(0, x, y)\right) d x d y<C_{1},
$$

and if the turbulent kinetic energy of $U$ and $V$ (defined as the $L_{2}$ norm of the fluctuation with respect to the Pouisseuille equilibrium profile) is less than $C_{2}$ for all time, i.e. $\forall t \geq 0$,

$$
\int_{0}^{1} \int_{-\infty}^{\infty}\left(u^{2}(t, x, y)+V^{2}(t, x, y)\right) d x d y<C_{2},
$$

then the $L_{2}$ norms of $\tilde{U}, \tilde{V}$ converge to zero:

$$
\lim _{t \rightarrow \infty} \int_{0}^{1} \int_{-\infty}^{\infty}\left(\tilde{U}^{2}(t, x, y)+\tilde{V}^{2}(t, x, y)\right) d x d y=0
$$

The meaning of this theorem is that, for a fully developed channel flow (whether laminar or turbulent), with a Reynolds number possibly above the critical value (the boundary of linear stability) but not too far above it, the observer is guaranteed to be convergent to the real velocity and pressure field, provided its initial estimates are not too far from the actual initial profile.

\section{An Output Feedback Design}

In a companion paper [7] a state feedback control law is presented for the Navier-Stokes 2D channel flow linearized around the Poisseuille equilibrium profile

$$
\begin{aligned}
u_{t}= & \frac{1}{R e}\left(u_{x x}+u_{y y}\right)+4 y(y-1) u_{x} \\
& +4(2 y-1) V-p_{x}, \\
V_{t}= & \frac{1}{R e}\left(V_{x x}+V_{y y}\right)+4 y(y-1) V_{x}-p_{y},
\end{aligned}
$$

with boundary conditions

$$
\begin{aligned}
u(t, x, 0) & =0, \\
V(t, x, 0) & =0, \\
u(t, x, 1) & =U_{c}(t, x), \\
V(t, x, 1) & =V_{c}(t, x),
\end{aligned}
$$

where $U_{c}, V_{c}$ are the actuation variables for tangential and normal velocity. In this section we complete the result presenting an output feedback law, which employs the same actuators but assume that the state is unknown except at the uncontrolled wall, where measurements of $V_{y}(x, 0)$ and $P(x, 0)$ are available.

The dynamic controller that stabilizes plant (59)-(64) is

$$
\begin{aligned}
U_{c}(t, x) & =\int_{0}^{1} \int_{-\infty}^{\infty} Q_{u}(x-\xi, \eta) \hat{u}(t, \xi, \eta) d \xi d \eta \\
V_{c}(t, x) & =h(t, x)
\end{aligned}
$$

where $h$ verifies the equation

$$
h_{t}=h_{x x}+g(t, x),
$$

where

$$
\begin{aligned}
g= & \int_{0}^{1} \int_{-\infty}^{\infty} Q_{V}(x-\xi, \eta) \hat{V}(t, \xi, \eta) d \xi d \eta \\
& +\int_{-\infty}^{\infty} Q_{0}(x-\xi)\left(u_{y}(t, \xi, 0)-\hat{u}_{y}(t, \xi, 1)\right) d \xi,(68)
\end{aligned}
$$


and where $\hat{V}$ and $\hat{u}$ are obtained from

$$
\begin{aligned}
\hat{u}_{t}= & \frac{1}{R e}\left(\hat{u}_{x x}+\hat{u}_{y y}\right)-\hat{p}_{x} \\
& +4 y(y-1) \hat{u}_{x}+4(2 y-1) \hat{V} \\
& -\frac{1}{R e} \int_{-\infty}^{\infty} Q_{1}(x-\xi, y)\left(u_{y}(\xi, 0)-\hat{u}_{y}(\xi, 0)\right) d \xi \\
& +\int_{-\infty}^{\infty} Q_{2}(x-\xi, y)(p(\xi, 0)-\hat{p}(\xi, 0)) d \xi \\
\hat{V}_{t}= & \frac{1}{R e}\left(\hat{V}_{x x}+\hat{V}_{y y}\right)-\hat{p}_{y}+4 y(y-1) \hat{V}_{x} \\
& -\frac{1}{R e} \int_{-\infty}^{\infty} \hat{V}_{y}(\xi, 0) \int_{-\infty}^{\infty} \chi(k) l(k, y, 0) \mathrm{e}^{2 \pi i k(x-\xi)} d k d \xi \\
& +\frac{1}{R e} \int_{-\infty}^{\infty} Q_{2}(x-\xi, y)\left(u_{y}(\xi, 0)-\hat{u}_{y}(\xi, 0)\right) d \xi \\
& +\int_{-\infty}^{\infty} Q_{1}(x-\xi, y)(p(\xi, 0)-\hat{p}(\xi, 0)) d \xi,
\end{aligned}
$$

the pressure $\hat{p}$ is governed by

$$
\hat{p}_{x x}+\hat{p}_{y y}=8(2 y-1) \hat{V}_{x},
$$

and the boundary conditions for the observer are

$$
\begin{aligned}
\hat{u}(t, x, 0)= & \hat{V}(t, x, 0)=0 \\
\hat{u}(t, x, 1)= & U_{c} \\
\hat{V}(t, x, 1)= & V_{c} \\
\hat{P}_{y}(t, x, 0)= & \frac{-1}{R e} \hat{U}_{x y}(t, x, 0) \\
\hat{P}_{y}(t, x, 1)= & \frac{-1}{\operatorname{Re}}\left(\hat{U}_{x y}(t, x, 1)-\left(V_{c}\right)_{x x}(t, x)\right) \\
& -\left(V_{c}\right)_{t}(t, x) .
\end{aligned}
$$

The kernels $Q_{u}, Q_{V}$ and $Q_{0}$ are defined as

$$
\begin{aligned}
Q_{u}= & \int_{-\infty}^{\infty} \chi(k) K(k, 1, \eta) \mathrm{e}^{2 \pi i k(x-\xi)} d k \\
Q_{V}= & \int_{-\infty}^{\infty} \chi(k) 16 \pi k i(2 \eta-1) \cosh (2 \pi k(1-\eta)) \\
& \times \mathrm{e}^{2 \pi i k(x-\xi)} d k \\
Q_{0}= & \int_{-\infty}^{\infty} \chi(k) \frac{2 \pi k i}{R e} \mathrm{e}^{2 \pi i k(x-\xi)} d k
\end{aligned}
$$

The function $K(k, y, \eta)$ appearing in (77) is a (complex valued) gain kernel defined as

$$
K(k, y, \eta)=\lim _{n \rightarrow \infty} K_{n}(k, y, \eta)
$$

where $K_{n}$ is recursively defined as ${ }^{3}$

$$
\begin{aligned}
K_{0}= & -\frac{R e}{3} \pi i k \eta\left(21 y^{2}-6 y(3+4 \eta)+\eta(12+7 \eta)\right) \\
& -2 \pi k \frac{\cosh (2 \pi k(1-y+\eta))-\cosh (2 \pi k(y-\eta))}{\sinh (2 \pi k)} \\
& +4 i \operatorname{Re} \eta(\eta-1) \sinh (2 \pi k(y-\eta)) \\
& -6 \eta i \frac{\operatorname{Re}}{\pi k}(1-\cosh (2 \pi k(y-\eta))),
\end{aligned}
$$

\footnotetext{
${ }^{3}$ This infinite sequence is convergent, smooth, and uniformly bounded over $(y, \eta) \in[0,1]^{2}$, and analytic in $k$.
}

and

$$
\begin{aligned}
K_{n}= & K_{n-1} \\
& -4 \pi k i R e \int_{y-\eta}^{y+\eta} \int_{0}^{y-\eta} \int_{-\delta}^{\delta}\left\{\frac{\sinh (\pi k(\xi+\delta))}{\pi k}\right. \\
& -(2 \xi-1)+2(\gamma-\delta-1) \cosh (\pi k(\xi+\delta))\} \\
& \times K_{n-1}\left(k, \frac{\gamma+\delta}{2}, \frac{\gamma+\xi}{2}\right) d \xi d \delta d \gamma \\
& +\frac{R e}{2} \pi i k \int_{y-\eta}^{y+\eta} \int_{0}^{y-\eta}(\gamma-\delta)(\gamma-\delta-2) \\
& \times K_{n-1}\left(k, \frac{\gamma+\delta}{2}, \frac{\gamma-\delta}{2}\right) d \delta d \gamma \\
& +2 \pi k \int_{0}^{y-\eta} \frac{\cosh (2 \pi k(1-\delta))-\cosh (2 \pi k \delta)}{\sinh (2 \pi k)} \\
& \times K_{n-1}(k, y-\eta, \delta) d \delta .
\end{aligned}
$$

The closed loop system verifies the following result.

Theorem 3: Suppose the system (59)-(64), (65)-(82), with gains $l, Q_{1}$ and $Q_{2}$ defined by (16)-(23) has a classical solution. Then, the equilibrium $\omega(x, y) \equiv V(x, y) \equiv 0$ is exponentially stable.

This Theorem follows from [7] where the state feedback result is presented, combined with the method for observer design explained in the previous sections.

\section{DISCUSSION}

The result was presented in $2 \mathrm{D}$ for ease of notation. Since 3D channels are spatially invariant in both streamwise and spanwise direction, it is straightforward to extend the design to 3D, just by applying the Fourier transform in both invariant directions. For the nonlinear observer, additional measurements of $w_{y}(x, 0)$ are required. The output feedback result can be extended to a 3D setting as well, with additional actuation in the spanwise direction. The result can be also be extended to periodic channel flow, 2D or 3D, substituting the Fourier transform with Fourier series, with all other expressions still holding.

\section{ACKNOWLEDGEMENT}

We thank Tom Bewley for numerous helpful discussions and expert advice.

\section{REFERENCES}

[1] B. Bamieh, F. Paganini and M.A. Dahleh, "Distributed control of spatially-invariant systems," IEEE Trans. Automatic Control, vol. 45, pp. 1091-1107, 2000.

[2] J. Hoepffner, M. Chevalier,T.R. Bewley and D.S. Henningson, "State estimation in wall-bounded flow systems. Part 1. Perturbed laminar flows," Joumal of Fluid Mechanics, to appear.

[3] P.J. Schmid and D.S. Henningson. Stability and Transition in Shear Flows, Springer, 2001.

[4] S. C. Reddy, P.J. Schmid, and D.S. Henningson, "Pseudospectra of the Orr-Sommerfeld operator," SLAM J. Appl. Math., vol. 53, no. 1, pp. $15-47,1993$.

[5] A. Smyshlyaev and M. Krstic, "Closed form boundary state feedbacks for a class of partial integro-differential equations," IEEE Transactions on Automatic Control, vol. 49, pp. 2185-2202, 2004.

[6] A. Smyshlyaev and M. Krstic, "Backstepping observers for parabolic PDEs," Systems and Control Letters, vol. 54, pp. 1953-1971, 2005.

[7] R. Vazquez and M. Krstic, "A closed-form feedback controller for stabilization of linearized Navier-Stokes equations: the 2D Poisseuille flow," 2005 Conference on Decision and Control, Sevilla. 